\title{
Diquark and triquark correlations in the deconfined phase of QCD
}

\author{
I. M. Narodetskii, Yu. A. Simonov, and A. I. Veselov \\ Institute of Theoretical and Experimental Physics, Moscow 117218
}

\begin{abstract}
We use the non-perturbative $Q \bar{Q}$ potential at finite temperatures derived in the Field Correlator Method to obtain binding energies for the lowest eigenstates in the $Q \bar{Q}$ and $Q Q Q$ systems $(Q=c, b)$. The three-quark problem is solved by the hyperspherical method. The solution provides an estimate of the melting temperature and the radii for the different diquark and triquark bound states. In particular we find that $J / \psi$ and $c c c$ ground states survive up to $T \sim 1.3 T_{c}$, where $T_{c}$ is the critical temperature, while the corresponding bottomonium states survive even up to higher temperature, $T \sim 2.3 T_{c}$.

PACS numbers: $12.38 \mathrm{Lg}, 14.20 \mathrm{Lq}, 25.75 \mathrm{Mq}$
\end{abstract}

\section{Introduction}

Diquark and triquark correlations in the quark-gluon plasma (QGP) are important for understanding the dynamics of heavy-ion collisions, processes in the early Universe and possibly the cores of the neutron stars [1]. Whether hadrons survive in the deconfined QGP is one of the key questions in QCD. In particular, the behavior of charmonia across the deconfinement transition has been suggested 1 as hard probes of the QGP. Above $T_{c}$, the critical temperature, most part of static $Q \bar{Q}$ interactions disappear that eventually implies a dissolution of heavy quarkonia bound states into the continuum. A suppression of heavy quarkonia production in heavy ion collisions is usually considered as an observed signal of deconfinement.

On quite general grounds it is expected that the $Q \bar{Q}$ interactions get modified by temperature. As it was first mentioned in Ref. [3] an important part of the static interaction survives at the temperature above transition and can support the bound $Q \bar{Q}$ states even after the deconfinement transition. Later existing of charmonia bound states at $T \geq T_{c}$ was confirmed on the lattice [4].

\footnotetext{
1 This effect was first first investigated in a phenomenological potential model taking into account the Debye screening [2].
} 
Most recently, in line with the study of Ref. [3], a new approach called the Field Correlator Method (FCM), was proposed to study dynamics of QGP, where the main emphasis was done on the nonperturbative vaccuum fields [5], [6], [7]. Let us summarize the basic formulation of the FCM as applied to finite T. The approach is based on the study of the quadratic field correlators $<\operatorname{tr} F_{\mu \nu}(x) \Phi(x, 0) F_{\lambda \sigma}(0)>(x$ is Euclidian), where $\Phi(x, 0)$ is the parallel transporter necessary to maintain gauge invariance. The Gaussian correlator is expressed in terms of two scalar functions, $D(x)$ and $D_{1}(x)$, which define the static potential between heavy quarks at $T=0$, the confinement part of the potential, $\sigma r$, of is expressed only in terms of $D(x)$ :

$$
\sigma=2 \int_{0}^{\infty} d \lambda \int_{0}^{\infty} d \tau D\left(\sqrt{\lambda^{2}+\tau^{2}}\right)
$$

At $T \geq T_{c}$ one should distinguish between electric and magnetic correlators $D^{E}(x)$, $D^{H}(x), D_{1}^{E}(x)$, and $D_{1}^{H}(x)$, and, correspondingly, between $\sigma^{E}$ and $\sigma^{H}$. It was argued in [8] and later confirmed on the lattice [9] that above the deconfinement region $D^{E}(x)$ and, correspondingly, $\sigma^{E}$ vanish, while the colorelectric correlator $D_{1}^{E}(x)$ and colormagnetic correlators $D^{H}(x)$ and $D_{1}^{H}(x)$ should stay unchanged at least up to $T \sim 2 T_{c}$. However, the correlators $D^{H}(x)$ and $D_{1}^{H}(x)$ do not produce static quark-antiquark potentials, they only define the spatial string tension $\sigma_{s}=\sigma^{H}$ and the Debye mass $m_{D} \propto \sqrt{\sigma_{s}}$ that grows with the temperature in the dimensionally reduced limit [10].

The main source of the quark-antiquark static interaction at $T \geq T_{c}$ originates from the correlator $D_{1}^{E}(x)[11]$

$$
V_{Q \bar{Q}}^{n p}(r, T)=\int_{0}^{1 / T} d \nu(1-\nu T) \int_{0}^{r} \lambda d \lambda D_{1}^{E(n p)}\left(\sqrt{\lambda^{2}+\nu^{2}}\right),
$$

that is responsible for bound states of quarks and gluons in the QGP .

The purpose of this Letter is to report our latest results of low lying charmonium and bottomonium as well as $c c c, b b b$ bound states above $T_{c}$, obtained from the use of this approach. The paper is organized as follows. In section 2. we introduce the static $Q \bar{Q}$ potential. In section 3. the numerical results for the ground states of $J / \psi$ and $\Upsilon$ are presented. In section 4 . we evaluate the masses of the three-quark $c c c$ and $b b b$ baryons. Our conclusions are given in section 5 . 


\section{The heavy quark-antiquark potential at $T \geq T_{c}$}

In the framework of the FCM, the finite temperature behavior of the static $Q \bar{Q}$ potential $V_{Q \bar{Q}}(r, T)$ at $T \geq T_{c}$ is given by

$$
V_{Q \bar{Q}}(r, T)=V_{Q \bar{Q}}^{p e r t}(r, T)+V_{Q \bar{Q}}^{n p}(r, T) .
$$

In Eq. (3) $V_{Q \bar{Q}}^{p e r t}(r, T)$ describes the interaction at short distances whereas $V_{Q \bar{Q}}^{n p}(r, T)$ is the long-distance potential. The short distance interaction is represented by the perutrbative one-gluon exchange potential that undergoes a Debye screening by the color charges of the QGP

$$
V_{Q \bar{Q}}^{\mathrm{pert}}(r, T)=-C_{F} \frac{\alpha_{s}}{r} \exp \left(-m_{D} r\right)
$$

where $C_{F}=4 / 3$ is the color factor, $m_{D}$ is the inverse of the Debye screening radius. This form of the modified Coulomb potential has been used in earlier works (see e.g. Ref. [12]) to specify the in-medium potential between heavy quarks and determine the dissociation points of different quarkonium states. The Debye mass $m_{D}$ in Eq. (44) can be written as $m_{D}=2.06 \sqrt{\sigma_{s}(T)}$, where $\sigma_{s}(T)$ is the spatial string tension due to chromomagnetic confinement. In what follows we use the results of Ref. [13] where the quantity $\sqrt{\sigma_{s}(T)}$ has been computed nonperturbatively up to two loops in the deconfined phase of QCD. For $n_{f}=0$ the Debye mass varies between $0.8 \mathrm{GeV}$ and $1.4 \mathrm{GeV}$, when $T$ varies between $T_{c}=275 \mathrm{MeV}$ and $2 T_{c}$.

The long-distance interaction $V^{n p}(r, T)$ requires theoretical assumptions about its shape. We follow Ref. [14], where the long distance nonperturbative $Q \bar{Q}$ potential was derived analytically from the analysis of the non-perturbative part of the correlator function $D_{1}^{E}(x)$ :

$$
D_{1}^{E(n p)}(x)=B \exp \left(-M_{0}|x|\right) /|x|
$$

In Eq. (5) the coefficient $B$ must be considered as functions of the physical temperature. In the confinement region

$$
B=2 C_{F} \alpha_{s}^{f} \sigma_{a d j} M_{0}=6 \alpha_{s}^{f} \sigma_{f} M_{0}
$$

$\alpha_{s}^{f}$ being the freezing value of the strong coupling constant in the confinement region, $\sigma_{f}$ is zero temperature string tension, and $M_{0}$ has the meaning of the lowest gluelump mass [15]. Taking $\alpha_{s}^{f}=0.6, \sigma_{f}=0.18 \mathrm{GeV}^{2}, M_{0}=0.9 \mathrm{GeV}$, we get $B=0.583 \mathrm{GeV}^{3}$. Above the critical temperature one substitutes $B \rightarrow \xi(T) B$, where the $T$-dependent constant

$$
\xi(T)=\left(1-0.36 \frac{M_{0}}{B} \frac{T-T_{c}}{T_{c}}\right)
$$




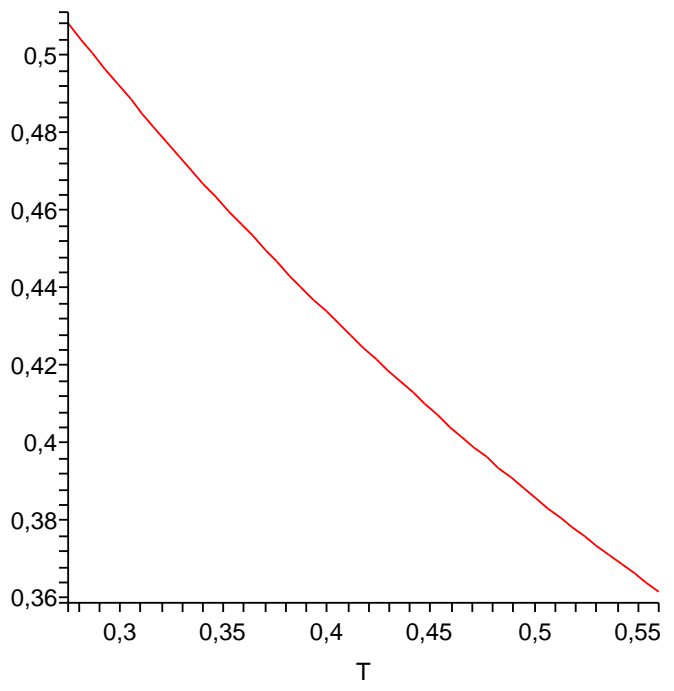

Figure 1: The dependence of the $V_{Q \bar{Q}}^{n p}(\infty, T)$ on the temperature $T$ given by Eq. (91).

arises from the comparison with lattice data [11]. As the result one obtains

$$
V_{Q \bar{Q}}^{n p}(r, T)=\xi(T)\left(V^{n p}(\infty, T)-V^{n p}(r)\right)
$$

where the continuum threshold (a constant shift in the potential) $V^{n p}(\infty, T)$ in Eq. (8) is

$$
V^{n p}(\infty, T)=\frac{B}{M_{0}^{2}}\left[1-\frac{T}{M_{0}}\left(1-\exp \left(-\frac{M_{0}}{T}\right)\right)\right]
$$

and

$$
V^{n p}(r)=\frac{B}{M_{0}^{2}} x K_{1}(x)+\mathcal{O}\left(\frac{T}{M_{0}}\right) .
$$

In Eq. (10) $K_{1}(x)$ is the modified Bessel function and $x=M_{0} r$. At $T=T_{c}$ one obtains $V_{Q \bar{Q}}^{n p}\left(\infty, T_{c}\right) \approx 0.5 \mathrm{GeV}$ that agrees with estimate obtained in Ref. [5] from lattice data. The large positive value of the continuum threshold is a consequence of non perturbative vaccuum fields and can not be explained in perturbation theory. The behavior of the potential $V_{Q \bar{Q}}(\infty, T)$ is shown in Fig. 1

\section{Quarkonia at finite temperature}

Having specified the static $Q \bar{Q}$ potential, we can now exploit the relativistic Hamiltonian technique, developed in [16] to calculate the masses of the $Q \bar{Q}$ states as a function of the temperature. This technique does not take into account chiral degrees of freedom and 
is applicable when spin-dependent interaction can be treated as perturbation. Therefore below consider heavy quarkonia and baryons. The masses of heavy quarkonia are defined as

$$
M_{Q \bar{Q}}=\frac{m_{Q}^{2}}{\mu_{Q}}+\mu_{Q}+E_{0}\left(m_{Q}, \mu_{Q}\right)
$$

where $E_{0}\left(m_{Q}, \mu_{Q}\right)$ is an eigenvalue of the Hamiltonian $H=H_{0}+V_{Q \bar{Q}}$, where $V_{Q \bar{Q}}$ is given by Eq. (3), $m_{Q}$ are the bare quark masses, and $\mu_{Q}$ are the constant auxiliary fields (AF) that were introduced to treat the kinematics of the relativistic particles. These parameters have to be found from the variational condition

$$
\frac{\partial M_{Q \bar{Q}}}{\partial \mu_{Q}}=0
$$

The eigenvalue problem is solved for each set of $\mu_{Q}$; then one has to minimize $M_{Q \bar{Q}}$ with respect to $\mu_{Q}$. Such an approach allows for a very transparent interpretation of AF: starting from bare quark masses $m_{Q}$, we naturally arrive at the dynamical masses $\mu_{Q}$ that appear due to the interaction. The AF are treated as c-number variational parameters. The bound state exists if $E_{0}\left(m_{Q}, \mu_{Q}\right) \leq V^{n p}(\infty, T)$.

To find out whether the non perturbative interaction can support the bound states at $T \sim T_{c}$ we use the Bargmann condition

$$
n \leq I=\mu_{Q} \int_{0}^{\infty}\left|V^{n p}(r)+V_{Q \bar{Q}}^{p e r t}(r, T)\right| r d r=\mu_{Q}\left(\frac{4 \alpha_{s}}{3 m_{d}}+\frac{B}{M_{0}^{4}}\right),
$$

where $n$ is the number of the $S$-wave bound states. Since $m_{d} \sim 1 \mathrm{GeV}, B / M_{0}^{2} \sim 0.5$ $\mathrm{GeV}$, and $M_{0} \sim 1 \mathrm{GeV}$, we conclude that to support at least one bound state one needs $\mu_{Q} \geq 1 \mathrm{GeV}$, i.e. there is no bound states of light quarks, but $c \bar{c}$ and especially $b \bar{b}$ binding is possible. Moreover, the bottomonium spectrum should display a much larger number of bound states above $T_{c}$.

The solutions for the binding energy for the $1 S$ states of charmonium and bottomonium are shown in Tables 1, 2. In these Tables we present the constituent quark masses $\mu_{Q}$ for $c \bar{c}$ and $b \bar{b} 2$, the differences $\varepsilon_{Q}=E_{0}-V_{Q \bar{Q}}(\infty)$, the mean squared radii $\sqrt{\overline{r^{2}}}$, and the masses $M_{Q \bar{Q}}$ for the $c \bar{c}$ and $b \bar{b}$ mesons. We employ $m_{c}=1.4 \mathrm{GeV}, m_{b}=4.8 \mathrm{GeV}$, and $\alpha_{s}=0.35$. As expected, we obtain the weakly bound state at $T=T_{c}$ that disappears at $T \sim 1.3 T_{c}$. The charmonium masses lie in the interval $3.2-3.3 \mathrm{GeV}$, that agrees with the results of Ref. [11]. Note that immediately above $T_{c}$ the mass of the $c \bar{c}$ state is about $0.2 \mathrm{GeV}$ higher than that of $J / \psi$.

\footnotetext{
${ }^{2}$ These masses are computed solely in terms of the bare quark masses $m_{c}$ and $m_{b}$, respectively. Note that, as in the confinement region [17, the constituent masses $\mu_{Q}$ only slightly exceed bare quark masses $m_{Q}$ that reflect smallness of the kinetic energies of heavy quarks.
} 
Table 1: The $1 S J / \psi$ state above the deconfinement region. $M_{0}=0.9 \mathrm{GeV}, B=0.583$ $\mathrm{GeV}^{3}, m_{c}=1.4 \mathrm{GeV}, \alpha_{s}=0.35$. Masses and energies are given in units of $\mathrm{GeV}$, the mean squared radius $\sqrt{\left.<r^{2}\right\rangle}$ in units of $\mathrm{GeV}^{-1}$.

\begin{tabular}{c|cccc}
\hline \hline$T / T_{c}$ & $\mu_{c}$ & $\varepsilon_{c}$ & $\sqrt{<r^{2}>}$ & $M_{c \bar{c}}$ \\
& & & & \\
\hline & & & & \\
1 & 1.451 & -0.019 & 7.53 & 3.291 \\
1.3 & 1.419 & +0.006 & 10.50 & 3.186 \\
& & & & \\
\hline \hline
\end{tabular}

Table 2: The $1 S \Upsilon$ state above the deconfinement region. The notations are the same as in Table 1, $m_{b}=4.8 \mathrm{GeV}$

\begin{tabular}{c|cccc}
\hline \hline$T / T_{c}$ & $\mu_{b}$ & $E_{0}-V_{Q \bar{Q}}(\infty)$ & $\sqrt{<r^{2}>}$ & $M_{b \bar{b}}$ \\
& & & & \\
\hline & & & & \\
1 & 4.984 & -0.300 & 1.27 & 9.815 \\
1.3 & 4.950 & -0.183 & 1.55 & 9.802 \\
1.6 & 4.915 & -0.095 & 2.06 & 9.783 \\
2.0 & 4.863 & -0.021 & 4.25 & 9.742 \\
2.2 & 4.832 & -0.003 & 7.62 & 9.712 \\
2.3 & 4.818 & +0.001 & 9.52 & 9.694 \\
& \multicolumn{5}{|c}{} \\
\hline \hline
\end{tabular}


As expected, the $b \bar{b}$ bound states remain intact up to the larger temperatures, $T \sim 2.3 T_{c}$, see Table 2. The masses of the $\mathrm{L}=0$ bottomonium lie in the interval 9.7-9.8 GeV, about $0.2-0.3 \mathrm{GeV}$ higher than $9.460 \mathrm{GeV}$, the mass of $\Upsilon(1 S)$.

The results for $c \bar{c}$ and $b \bar{b}$ bound states found from the FCM can be compared to the calculations based on phenomenological $Q \bar{Q}$ potentials identified with the free energy measured on the lattice [18], [19]. Our results for $1 S(J / \psi)$ are qualitatively agree with those of Refs. [18], [19] while our melting temperature for $1 S(\Upsilon)$ is much smaller than $T \sim(4-6) T_{c}$ found in Ref. [19].

\section{4. $Q Q Q$ baryons at $T \geq T_{c}$}

The three quark potential is given by

$$
V_{Q Q Q}=\frac{1}{2} \sum_{i<j} V_{Q \bar{Q}}\left(r_{i j}, T\right)
$$

where $\frac{1}{2}$ is the color factor. We solve the three quark Schrödinger equation using the hyperspherical method. The wave function of a $Q Q Q$ baryon depends on the three-body Jacobi coordinates

$$
\boldsymbol{\rho}_{i j}=\sqrt{\frac{\mu_{Q}}{2}}\left(\boldsymbol{r}_{i}-\boldsymbol{r}_{j}\right), \quad \boldsymbol{\lambda}_{i j}=\sqrt{\frac{2}{3} \mu_{Q}}\left(\frac{\boldsymbol{r}_{i}+\boldsymbol{r}_{j}}{2}-\boldsymbol{r}_{k}\right),
$$

$(i, j, k$ cyclic). There are three equivalent ways of introducing the Jacobi coordinates, which are related to each other by linear transformations with the Jacobian equal to unity. In what follows we omit the indices $i$ and $j$.

In terms of the Jacobi coordinates the three-quark kinetic energy operator $H_{0}$ is written as

$$
H_{0}=-\frac{1}{2}\left(\frac{\partial^{2}}{\partial \boldsymbol{\rho}^{2}}+\frac{\partial^{2}}{\partial \boldsymbol{\lambda}^{2}}\right)=-\frac{1}{2}\left(\frac{\partial^{2}}{\partial R^{2}}+\frac{5}{R} \frac{\partial}{\partial R}+\frac{\boldsymbol{L}^{2}(\Omega)}{R^{2}}\right),
$$

where $R$ is the six-dimensional hyperradius that is invariant under quark permutations,

$$
\begin{aligned}
& R^{2}=\rho^{2}+\lambda^{2}=\frac{\mu_{Q}}{3}\left(r_{12}^{2}+r_{23}^{2}+r_{31}^{2}\right), \\
& \rho=R \sin \theta, \quad \lambda=R \cos \theta, \quad 0 \leq \theta \leq \pi / 2,
\end{aligned}
$$

$\Omega$ denotes five residuary angular coordinates, and $\boldsymbol{L}^{2}(\Omega)$ is an angular operator

$$
\mathbf{L}^{2}=\frac{\partial^{2}}{\partial \theta^{2}}+4 \cot \theta \frac{\partial}{\partial \theta}-\frac{\mathbf{l}_{\rho}^{2}}{\sin ^{2} \theta}-\frac{\mathbf{l}_{\lambda}^{2}}{\cos ^{2} \theta},
$$

whose eigenfunctions (the hyperspherical harmonics) satisfy

$$
\mathbf{L}^{2}(\Omega) Y_{[K]}\left(\theta, \mathbf{n}_{\rho}, \mathbf{n}_{\lambda}\right)=-K(K+4) Y_{[K]}\left(\theta, \mathbf{n}_{\rho}, \mathbf{n}_{\lambda}\right)
$$


with $K$ being the grand orbital momentum.

The wave function $\psi(\boldsymbol{\rho}, \boldsymbol{\lambda})$ is written in a symbolical shorthand as

$$
\psi(\boldsymbol{\rho}, \boldsymbol{\lambda})=\sum_{[K]} \psi_{[K]}(R) Y_{[K]}(\Omega)
$$

where the set $[K]$ is defined by the orbital momentum of the state and the symmetry properties. We truncate this set using the hypercentral approximation $K=K_{\min }=0$. Introducing the reduced function $u(R)$

$$
\Psi(\boldsymbol{\rho}, \boldsymbol{\lambda}, T)=\frac{1}{\sqrt{\pi^{3}}} \frac{u(R, T)}{R^{5 / 2}},
$$

and averaging the three-quark potential (14) over the six-dimensional sphere one obtains the one-dimensional Schrödinger equation for $u(R, T)$

$$
\frac{d^{2} u(R, T)}{d R^{2}}+2\left[E_{0}-\frac{15}{8 R^{2}}-\frac{3}{2} \xi(T)\left(\mathcal{V}^{\text {pert }}(R, T)+\mathcal{V}^{n p}(R, T)\right)\right] u(R, T)=0
$$

where

$$
\begin{aligned}
& \mathcal{V}^{\text {pert }}(R, T)=-\frac{4}{3} \alpha_{s} \frac{a_{C}(R)}{R}, \\
& a_{C}(R)=\frac{16}{\pi} \sqrt{\frac{\mu_{Q}}{2}} \int_{0}^{\pi / 2} \exp \left(\frac{-m_{D} R \sin \theta}{\sqrt{\mu_{Q} / 2}}\right) \sin \theta \cos ^{2} \theta d \theta,
\end{aligned}
$$

and

$$
\mathcal{V}^{\mathrm{np}}(R, T)=V^{n p}(\infty, T)-\frac{16 B}{\pi M_{0}} \sqrt{\frac{2}{\mu_{Q}}}\left(\int_{0}^{\pi / 2} K_{1}\left(\frac{M_{0} R \sin \theta}{\sqrt{\mu_{Q} / 2}}\right) \sin ^{3} \theta \cos ^{2} \theta d \theta\right) R
$$

The temperature dependent mass of the colorless $Q Q Q$ states is defined as

$$
M_{Q Q Q}=\frac{3}{2} \frac{m_{Q}^{2}}{\mu_{Q}}+\frac{3}{2} \mu_{Q}+E_{0}\left(m_{Q}, \mu_{Q}\right) .
$$

The bound $Q Q Q$ state exists if $E_{0}\left(m_{Q}, \mu_{Q}\right) \leq V_{Q Q Q}(\infty, T)$, where

$$
\mathcal{V}_{Q Q Q}(\infty, T)=\frac{3}{2} V^{n p}(\infty, T)
$$

In Tables 3 and 4 we show the masses of the ground $c c c$ and $b b b$ states as a function of the temperature. 
Table 3: The ground $c c c$ state as a function of the temperature above the deconfinement region. The notations are the same as in Table 1

\begin{tabular}{c|cccc}
\hline \hline$T / T_{c}$ & $\mu_{c}$ & $E_{0}-\mathcal{V}_{\mathcal{Q Q} \mathcal{Q}}(\infty)$ & $\sqrt{<R^{2}>}$ & $M_{c c c}$ \\
& & & & \\
\hline & & & & \\
1 & 1.474 & -0.046 & 6.60 & 4.922 \\
1.3 & 1.434 & -0.002 & 9.96 & 4.769 \\
& & & & \\
\hline \hline
\end{tabular}

Table 4: The ground $b b b$ state as a function of the temperature above the deconfinement region. The notations are the same as in Table 1

\begin{tabular}{c|cccc}
\hline \hline$T / T_{c}$ & $\mu_{b}$ & $E_{0}-\mathcal{V}_{\mathcal{Q Q} \mathcal{Q}}(\infty)$ & $\sqrt{<R^{2}>}$ & $M_{b b b}$ \\
& & & & \\
\hline & & & 3.12 & 14.665 \\
1 & 4.977 & -0.506 & 3.86 & 14.661 \\
1.3 & 4.927 & -0.314 & 5.04 & 14.645 \\
1.6 & 4.885 & -0.171 & 8.21 & 14.598 \\
2.0 & 4.840 & -0.046 & 10.59 & 14.562 \\
2.2 & 4.822 & -0.011 & 11.62 & 14.541 \\
2.3 & 4.815 & +0.001 & & \\
\hline \hline
\end{tabular}




\section{Conclusions}

Based on the non-perturbative dynamics driven by the field correlators at finite temperatures we have calculated binding energies for the lowest eigenstates in the $c \bar{c}, c c c, b \bar{b}$, and $b b b$ systems. For what concerns the charmonium we find that $J / \psi$ (and $c c c$ ground states) survive up to $T \sim 1.3 T_{c}$. On the other hand, the $b \bar{b}$ and $b b b$ states survive even up to higher temperature, $T \sim 2.3 T_{c}$. This suggests that the systems are strongly interacting above $T_{c}$.

This work was supported in part by RFBR Grants \# 08-02-00657, \# 08-02-00677, \# 09-02-00629 and by the grant for scientific schools \# NSh.4961.2008.2.

\section{References}

[1] B. Müller and J. L. Nagle, Ann. Rev. Nucl. Part. Sci. 56, 93 (2006) ; K. Nagi, T. Hatsuda, and Y. Miake, Quark-Gluon Plasma, Cambridge University Press, Canbridge 2005

[2] T. Matsui and H. Satz, Phys. Lett. B178 (1986) 416

[3] Yu. A. Simonov, JETP Lett. 54, 249 (1991); Phys. At. Nucl. 58, 309 (1995)

[4] I. Wetzorke, F. Karsch, E. Laermann, P. Petreczky, and S.Stickan, Nucl. Phys. Proc. Suppl. 106, 510 (2002); P.Petreczky, arXiv: hep-lat/0409139, hep-lat/0502008. K.Hübner, O.Kaczmarek, F.Karsch, O.Vogt, arXiv: hep-lat/0408031. M. Asakawa and T. Matsuda, Phys. Rev. Lett. 92, 012001 (2004)

[5] Yu. A. Simonov, M. A. Trusov, Phys. Lett. B 650, 650 (2007)

[6] Yu. A. Simonov, Ann. Phys. 323, 783 (2008), E. V. Komarov and Yu. A. Simonov, Ann. Phys. 323, 1230 (2008)

[7] A. V. Nefediev, Yu. A. Simonov, M. A. Trusov, Int. J. Mod. Phys. E18, 549 (2009), arXiv: hep-ph/0902.0125

[8] Yu. A. Simonov, JETP Lett. 55, 605 (1992), Phys.Lett. B 619, 293 (2005) .

[9] M. DElia, A. DiGiacomo, and E. Meggiolaro, Phys. Rev. D67, 114504 (2003)

[10] N. O. Agasian and Yu. A. Simonov, Phys. Lett. B639, 82 (2006)

[11] A. DiGiacomo, E. Meggiolaro, Yu. A. Simonov, and A. I. Veselov, Phys. Atom. Nucl. 70, $908(2007)$ 
[12] S. Digal, P. Petreczky, and H. Satz, Phys. Rev. D64, 094015 (2001)

[13] N. O. Agasian, Phys. Lett. B 562, 257 (2003), arXiv:hep-ph/0303127.

[14] Yu. A. Simonov, Phys. Lett. B619, 293 (2005)

[15] Yu. A. Simonov, Nucl. Phys. B592 350 (2001), arXiv: hep-ph/0003114.

[16] A. Yu. Dubin, A. B. Kaidalov, and Yu .A. Simonov, Phys. Lett. 323, 4 (1994)

[17] I. M. Narodetskii, M. F. Trusov, A. I. Veselov, Phys. Atom. Nucl. 72, 536 (2009), arXiv: hep-ph/0801.1980

[18] D. Blaschke, O. Kaczmarek, E. Laermann, V. Yudichev, Eur. Phys. J. C43, 81 (2005)

[19] W. M. Alberico, A. Beraudo, A. De Pace, and A. Molinari, arXiv: hep-ph/0507084 